\title{
Éduquer dans un monde pluriel : François Dubet (entretien avec Geneviève Zoïa)
}

\section{François Dubet}

\section{(2) OpenEdition}

1 Journals

\section{Édition électronique}

URL : http://journals.openedition.org/trema/2697

DOI : 10.4000/trema.2697

ISSN : 2107-0997

\section{Éditeur}

Faculté d'Éducation de l'université de Montpellier

\section{Édition imprimée}

Date de publication : 1 avril 2012

Pagination : $6-21$

ISSN : 1167-315X

\section{Référence électronique}

François Dubet, "Éduquer dans un monde pluriel : François Dubet (entretien avec Geneviève Zoïa)», Tréma [En ligne], 37 | 2012, mis en ligne le 01 avril 2014, consulté le 02 mai 2019. URL : http:// journals.openedition.org/trema/2697 ; DOI : 10.4000/trema.2697

Ce document a été généré automatiquement le 2 mai 2019.

Trema 


\title{
Éduquer dans un monde pluriel : François Dubet (entretien avec Geneviève Zoïa)
}

\author{
François Dubet
}

1 L'école laïque "d'avant" est souvent décrite comme habitée par des combattants héroïques de la laïcité, intransigeants et militants. On se la représente même comme un espace garant de la mixité sociale et de l'égalité, par contraste avec une neutralité aujourd'hui mise à mal par les ghettos, la ségrégation, le communautarisme. Est-ce le reflet de la réalité ? Comment décrire cette ancienne école laïque ?Était-elle en réalité si militante et anticléricale? Quid des minorités de l'époque, les cultures locales et les autres religions? Comment fonctionnait ce modèle magique que vous avez décrit?

Si l'école laïque républicaine a été combattante, ce fut d'abord contre l'église

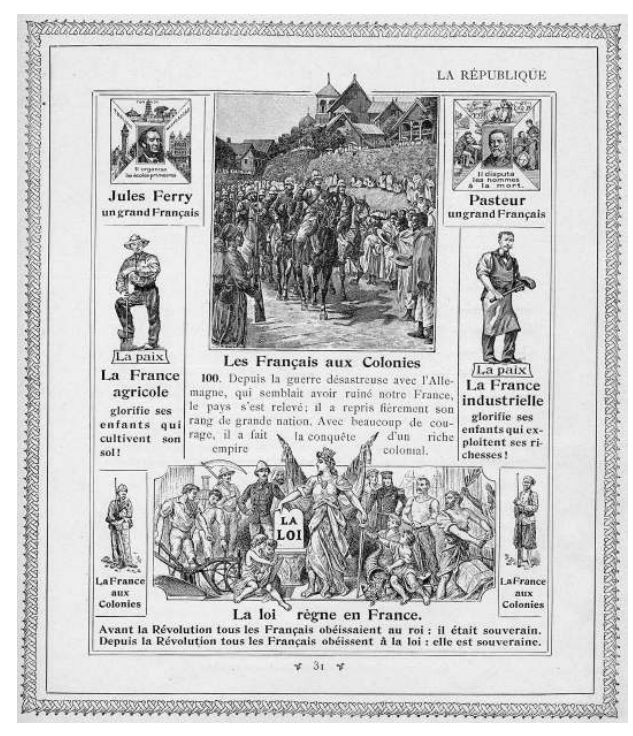
en tant qu'institution. Cet anticléricalisme dur, répondant d'ailleurs à une Église hostile à la République et à la démocratie, n'a pas toujours été aussi anti religieux que le suppose la légende des hussards noirs. On sait que la plupart des institutrices avaient une pratique religieuse régulière avant la guerre de 1914. Le calendrier scolaire était, et reste, un compromis avec les fêtes religieuses et la morale laïque visait à ne pas heurter la morale religieuse ; elle était, elle aussi, rigoureuse et traditionnelle, elle séparait les sexes, elle était patriotique et défendait la famille... En se repliant sur le thème de l'instruction, l'école laïque ne se mêlait pas directement des croyances et les historiens ont montré que la baisse de la pratique religieuse dans un grand nombre de départements a été très antérieure aux lois de Jules FERRY. Il reste que 
l'école laïque s'est faite contre l'Église, mais dans un monde social où le catholicisme était une sorte d'évidence culturelle, y compris pour les adversaires de l'Église.

Pour ce qui est de son recrutement social, l'école républicaine de la troisième, puis de la quatrième République, fut sans doute moins mixte et plus mixte que ne l'est l'école d'aujourd'hui. Elle était nettement moins mixte en ce qui concerne les cycles scolaires. L'école républicaine, c'était d'abord l'école élémentaire dans laquelle se retrouvaient tous les enfants du peuple. Les enfants de la bourgeoisie allaient au petit lycée payant et le collège était ouvert aux classes moyennes et aux meilleurs élèves des catégories populaires. Quant au lycée et à l'université, ils étaient largement réservés à la bourgeoisie dans un système profondément malthusien: $2 \%$ d'une classe d'âge obtenait le baccalauréat en 1900, 6\% en 1945. Au fond, cette école était organisée comme une école de classes (de classes sociales) où seuls quelques boursiers échappaient à leur destin social et scolaire sans que cette situation soit perçue comme un scandale par la grande majorité de la population jusqu'aux années 1950.

4 Il est vrai que cette école, notamment l'école élémentaire, a été vécue comme un formidable progrès de l'égalité et de la mixité sociale puisque tous les enfants du peuple, tous les enfants du même village ou du même quartier, partageaient les mêmes bancs de classes bien que la mixité scolaire ait été pondérée par la formation de classes de niveaux, classes fortes et classes faibles, et par un traitement assez contrasté des bons élèves et des cancres. Parce que l'école républicaine était « naturellement » inégalitaire, parce que l'on y croyait aux "dons» et aux destins sociaux dans une société tolérant mieux les inégalités qu'aujourd'hui, les scolarités étaient peu concurrentielles puisque les diplômes, rares, jouaient un rôle limité dans les parcours sociaux du plus grand nombre.

L'image enchantée et nostalgique de cette école républicaine tient à la rupture qu'elle a introduite avec le monde social antérieur: tous les enfants allaient enfin à l'école, la plupart dans la même école pour y apprendre les mêmes choses sanctionnées par le même certificat d'études primaires... Mais si cette école ouvrait l'accès à une culture et à une langue communes (n'oublions pas que de nombreux Français parlaient, soit des patois, soit d'autres langues comme le basque, le breton, l'occitan), elle pesait relativement peu sur le destin social de la plupart des élèves, à l'exception d'une minorité de boursiers qui s'élevaient dans la société grâce à l'école et qui en forgeront la légende heureuse.

5 Aujourd'hui, la mixité sociale à l'école est objectivement plus forte avec le collège unique et l'ouverture du lycée et de l'enseignement supérieur à la majorité des élèves et donc à beaucoup d'élèves issus de milieux modestes. Mais cette ouverture, cette massification, a entraîné une diversification interne au système sous l'effet d'une distinction continue et d'une concurrence latente entre les élèves, les familles, les établissements, les filières, les diplômes... $65 \%$ des élèves obtiennent le bac, mais tous les bacs ne se valent pas, les diplômes sont accessibles, mais leurs valeurs sont profondément inégales, les filières et les établissements se distinguent de plus en plus.

Les familles jouent le jeu de la distinction scolaire par la fuite des établissements et des écoles les plus difficiles. Le recrutement social des élites scolaires s'est resserré pendant que celui des élèves en difficulté reste toujours aussi marqué socialement. Et comme tous ces effets sont contradictoires avec les promesses de la massification démocratique, nous avons le sentiment que la mixité sociale décline profondément parce que là où il y avait une volonté d'unité, malgré une sorte d'apartheid social et scolaire, se déploie une volonté de séparation, une obsession de la concurrence au sein même d'une école dont la 
structure inégalitaire a été progressivement résorbée. Nous avons donc toutes les raisons d'être déçus par la situation actuelle, à condition de ne pas idéaliser le passé.

La laïcité en France ne fut pas seulement un principe rigide ou une règle stricte d'indifférence et de neutralité dans le cadre scolaire. Peut-on préciser en quoi elle inspira pratiquement les expériences scolaires des élèves et des maitres, les organisations, les programmes, règles, l'environnement? La laïcité a été l'instrument d'un projet éducatif et politique puissant pour la construction d'une culture commune appuyée sur une raison pensée comme universelle et la construction d'une nation moderne. Aujourd'hui, des discours invoquent les demandes identitaires pour expliquer une crise de la laïité et du modèle universaliste républicain. Peut-on ainsi opposer universalisme et particularisme? Les difficultés que connaît la laïcité aujourd'hui proviennent d'un mouvement profond d'évolution de l'école. Quelles transformations sociales majeures ont affecté le principe de laïcité tel qu'il est associé à l'école républicaine d'antan? Justifier le déclin de la laïcité par la montée des particularismes ne revient-il pas à s'accrocher à une immuable laïité de surplomb, alors que l'école et la société changent?

7 De manière assez classique, il est possible de définir la laïcité de deux manières. La première serait une laïcité prudente ou «négative » pour reprendre le mot de Benjamin CONSTANT sur la démocratie. La laïcité scolaire est une règle de séparation et de tolérance. Séparation de la religion et de l'éducation impliquant ce que Jules FERRY attendait des maîtres d'école : que leurs propos ne puissent jamais choquer les croyances des familles. Tolérance exigeant que tous les élèves soient accueillis quelles que soient leurs croyances. Cette laïcité-là exigeait des mœurs scolaires prudentes et mesurées et l'enseignement d'une morale suffisamment partagée pour que personne n'y trouve à redire. La généalogie de cette laïcité modeste doit beaucoup aux héritiers des Lumières, mais elle doit aussi aux catholiques éclairés et surtout aux juifs et aux protestants qui attendaient de l'école publique qu'elle les protège du prosélytisme et de la volonté de puissance de l'Église. Je crois qu'il n'y a rien à redire à cette forme de laïcité entraînant une certaine distance de l'école à l'égard des diverses demandes sociales. Il me semble d'ailleurs qu'elle n'est guère remise en cause.

8 La laïcité de la troisième République a eu un visage plus offensif en promouvant des valeurs républicaines capables de fonder un nouvel accord politique et culturel autour de la nation et de la croyance dans le règne de la Raison et du Progrès, autour d'un universel alternatif à l'universel religieux. C'est la laïcité combattante dont quelques groupes militants sont encore les porte-paroles mais dont les forces ont longuement décliné au fil de la seconde moitié du XXe siècle quand il était clair que l'Église et la droite dure avaient accepté la République après le déshonneur de la collaboration. La laïcité offensive devenait prudente, surtout après la défaite des «ultras » laïques de 1983 et une mise à distance des cadres religieux de l'école privée accueillant les catholiques et tous les autres, croyants ou pas. En tous cas, la guerre laïque semblait éteinte à jamais.

9 Tout change avec l'installation en France de nouvelles religions, notamment de l'Islam. Ce n'est pas que l'Islam, à l'exception de quelques groupes radicaux, remette en cause le principe même de la laïcité, mais l'Islam déstabilise un universalisme laïque qui n'est pas aussi universel qu'on le pensait «naïvement». Il apparait que la laïcité française est nationale, chrétienne, qu'elle fut liée au colonialisme et qu'elle doit aujourd'hui faire une place à des identités et à des cultures qui, non seulement lui sont étrangères, mais qu'elle a dominées. Bien plus que par une demande religieuse "hétérogène ", la laïcité est menacée par le fait que nous entrons dans des sociétés nationales pluriculturelles. Alors que la laïcité française a été associée au triomphe d'un modèle national se voulant 
universel, on s'aperçoit, partout ou presque partout, que les sociétés nationales sont de plus en plus diverses et que les migrants n'ont pas vocation à perdre toutes leurs racines et leurs cultures en devenant des citoyens nationaux. Dès lors, il est évident que l'universalisme laïque participant partiellement d'un particularisme national, il faut redéfinir et renégocier la laïcité, redéfinir les relations entre ce qui est vu comme un particularisme et ce qui est conçu comme universel.

Par exemple, nous avons assez rapidement pensé que la tolérance laïque ne pouvait pas imposer la consommation de porc à tous les élèves. Ce qui est plutôt un progrès par rapport aux périodes antérieures. On sait que la présence du foulard religieux est refusée au nom d'un principe laïque vécu comme universel, c'est-à-dire non négociable, à savoir l'égalité entre les sexes. Quelles que soient les positions que l'on puisse avoir sur ces thèmes, et qu'elles concernent les demandes musulmanes ou d'autres demandes, il me semble clair que le principe de tolérance doit être maintenu jusqu'au moment où les demandes menaceraient ce principe de tolérance lui-même. Peut-être pourrions-nous adopter en la matière la position défendue par Will KIMLICKA au Canada (KIMLICKA, 2001) : toutes les revendications culturelles sont légitimes dès lors qu'elles ne portent pas atteinte à la liberté de conscience, à l'égalité et l'autonomie des individus. On peut porter un «foulard " à l'école quand on est assuré que la personne qui le porte a fait ce choix, qu'elle ne refuse pas que d'autres fassent d'autres choix et qu'elle ne refuse pas de fréquenter les mêmes classes que les autres. C'est d'ailleurs ce que nous faisons à l'université, où l'on considère que tous les étudiants, et toutes les étudiantes, sont responsables d'eux-mêmes.

11 Cependant les débats sont d'autant plus difficiles que nous ne vivons pas dans un monde de pure philosophie. Il me semble évident qu'une laïcité rigide masque mal un rejet pur et simple des migrants et des étrangers, alors qu'il est tout aussi évident que l'appel à une tolérance laïque élargie masque parfois tout aussi mal les pires intolérances religieuses. À des demandes ethniques s'oppose une laïcité française tout aussi ethnique. Il m'arrive de penser que l'extrême droite et la critique postcoloniale des Indigènes de la République fonctionnent de manière symétrique inverse en assignant les individus à des identités historiques intangibles.

12 Si nous voulons promouvoir une laïcité ouverte, nous n'échapperons pas à une nouvelle casuistique laïque et la sagesse politique devrait d'abord s'interdire d'instrumentaliser ce débat. On peut être inquiet à ce propos. Il suffit de voir comment la défense la plus vigoureuse de laïcité a basculé vers l'extrême droite et vers une partie de la droite dure pour avoir les plus grandes suspicions en la matière : la défense de la laïcité est alors le masque du vieux nationaliste chauvin.

Les rapports sociaux dans les établissements scolaires des quartiers en difficulté sociale sont souvent interprétés en termes ethniques et on identifie spontanément l'immigration qui caractérise ces quartiers aux difficultés scolaires. Ce raisonnement impute l'échec à des mécanismes externes à l'école, comme les socialisations populaires par exemple, soit en terme déficitaire, soit en termes de différence. De fait, la question de la différence culturelle se pose essentiellement en France dans le cadre de rapports sociaux inégalitaires, et ainsi la revendication identitaire ne semble se manifester que sous l'angle de la crise de l'institution et de la laïcité: régimes alimentaires, demandes d'aménagement de l'emploi du temps, évitement de certaines disciplines, conflits de vie scolaire, façons de parler, ethnicisation des interactions sociales à l'école... Dès lors, ne peut-on pas parler d'un retournement du stigmate plutôt que de revendications culturelles? En dehors des tensions liées à l'échec scolaire ou la difficulté sociale, on entend peu parler de différence culturelle 
à l'école et de laïcité. Le brouillage entre cultures et stigmates risque-t-il d'entretenir un racisme plus ou moins déclaré?

De manière générale, à l'école comme ailleurs, on assiste à une « ethnicisation » ou à une « racialisation » des problèmes sociaux. Ceci tient au processus urbain de concentration des populations étrangères ou issues de l'immigration dans les mêmes quartiers et dans les mêmes écoles. Ce phénomène est si intense à la périphérie des grandes villes et dans certaines zones centrales dégradées que l'on ne voit plus ces groupes comme la classe ouvrière ou comme les classes populaires, mais comme des étrangers et des immigrés alors même que, bien souvent, il ne s'agit plus d'immigrés proprement dit. C'est ainsi que les acteurs sont représentés et, il faut bien le dire, qu'ils se représentent et s'identifient eux-mêmes. Dans les couches les moins favorisées de la société, la structure « raciale »vue comme raciale - recouvre la structure sociale - vue comme sociale. Dès lors, ce que l'on interprétait comme des problèmes strictement sociaux, au point de ne pas voir les migrants, est interprété comme des problèmes culturels et ethniques, au point de ne plus voir les classes sociales.

Cette évolution, une des plus spectaculaires des trente dernières années, se décline aussi à l'école. Le symbole de l'élève en échec n'est plus le jeune prolétaire appelé à entrer à l'usine; c'est le jeune, surtout un garçon, "issu de l'immigration" même quand il appartient à la troisième génération. Avec ce changement, l'étiologie spontanée de l'échec scolaire se transforme profondément. Au thème de la distance sociale entre l'école «bourgeoise " et la classe ouvrière, se substitue celui de la distance culturelle entre l'école républicaine et les populations venues d'ailleurs. En fait, on change de cadre de lecture des problèmes scolaires. L'inégalité sociale n'est plus perçue en termes de domination économique, de domination de classes, elle est perçue en termes de "différences » culturelles, «de races». Les acteurs critiques, souvent des sociologues, suivent ce changement de catégories : l'école «bourgeoise et capitaliste » est remplacée par l'école "républicaine postcoloniale »; là où on parlait d'inégalités, on parle de discriminations tout en désignant souvent strictement les mêmes « faits ».

Ce changement de paradigme de la perception des problèmes scolaires ne signifie pas que les problèmes changent « vraiment ». On sait par exemple que les élèves les plus distants culturellement de l'école ne sont pas forcément ceux qui éprouvent les plus grandes difficultés scolaires; il n'est pas rare que des primo-migrants réussissent mieux que des deuxièmes ou troisièmes générations. On sait aussi que l'appartenance aux catégories sociales favorisées efface les effets supposés négatifs de la distance culturelle. Mais on sait surtout que les élèves supposés « différents » ne le sont pas tant que ça. Dans le processus de ghettoïsation que nous observons, il se crée plutôt une culture juvénile anti-scolaire alors même que les parents, culturellement plus « différents », ont une grande confiance dans l'école. La concentration spatiale des problèmes sociaux dégradant profondément les conditions d'éducation des quartiers définis comme « difficiles » - ce qui prouve à quel point les classes moyennes font la norme - ouvre un cercle vicieux d'accentuation des difficultés bien mis en évidence par John OGBU aux États-Unis: les jeunes noirs des ghettos pensent que l'école n'est pas faite pour eux, voire qu'elle est faite contre eux. Autour de ces écoles s'organisent des mécanismes que vous avez bien étudiés avec Laurent VISIER : il faut fuir les établissements les plus faibles et le nombre d'élèves issus de l'immigration est tenu pour un bon indicateur de la faiblesse de ces établissements. Ce choix a priori rationnel, bien plus que l'expression d'une haine raciale, engendre la ghettoïsation scolaire que chacun condamne. On commencerait à observer aujourd'hui la 
fuite des élèves d'origine maghrébine devant l'afflux des élèves venus d'Afrique Noire parce que ceux-ci feraient baisser le niveau. À terme, les élèves ainsi "racialisés " retournent le stigmate, rejettent l'institution, affirment une «différence » qui n'est le plus souvent qu'une inégalité sociale.

17 Il n'empêche que ces processus ne sont pas de purs fantasmes parce qu'ils ont des effets de réalité extrêmement solides en termes de stratégies des acteurs, de création de dispositifs politiques, de productions théoriques, de modèles politiques et, au bout du compte, de formation de la subjectivité même des individus. Dès lors, les questions culturelles prennent leur propre autonomie puisque c'est à travers elles que les uns et les autres interprètent et comprennent leurs expériences.

Pourquoi la question de la différence culturelle vient-elle frapper à la porte de l'école? Comment le sujet entre t-il à l'école, avec ses différences et ses exigences en matière de respect, de justice, de considération?

19 J'ai le sentiment que le thème de la différence et que la demande de reconnaissance des différences à l'école sont nettement moins présents que celui de la demande de justice sociale et de la justice scolaire. Si l'on se place du côté des élèves et de leurs familles, le discours quasi hégémonique est celui des espérances et de la déception face à un système scolaire qui ne semble pas capable de tenir ses promesses de réussite et d'égalité. Je n'ai jamais entendu des élèves dire que leur échec tenait à la manière dont les programmes scolaires parlaient des croisades, des religions monothéistes et de l'histoire coloniale. En revanche, il nous est dit sans cesse que la concentration de toutes les difficultés scolaires et sociales dans les mêmes établissements est une injustice de base. Plus encore, les élèves dénoncent les discriminations rencontrées lors des stages, à l'entrée sur le marché du travail : on parle plus des diplômés chômeurs que du racisme des enseignants. Dans une enquête en cours portant sur l'expérience des discriminations (O. COUSIN, F. DUBET, E. MACÉ et S. RUI) n'émergent guère de demandes de reconnaissance des différences culturelles à l'école et on peut se demander si celles-ci ne sont pas une des constructions idéologiques et politiques qui se projettent aujourd'hui sur l'école.

Quant aux demandes de respect, de justice et de considération, elles sont portées pas tous les élèves confrontés à la tension entre l'idéal de l'égalité de tous et les hiérarchies scolaires. Le besoin de respect est une manière d'exiger que l'égalité et la dignité de chaque élève soit assurée alors que l'école a pour «fonction » de produire des inégalités tout en affirmant l'égalité fondamentale de tous les élèves. Pour le dire vite, je n'ai pas le sentiment que la demande de justice soit nécessairement associée à une protestation contre le déni des différences. Au contraire, elle est plutôt un appel à la ressemblance chez des élèves qui ne voient leurs différences que sous la forme d'un stigmate. Les élèves sont plus sensibles à la " discrimination négative » qu'aux rêves d'une école orientée vers la défense des cultures qui ne sont souvent que des bribes et des reconstructions. D'ailleurs la culture scolaire est avant tout scolaire et elle n'est jamais le prolongement des diverses cultures sociales au sein du même cadre national. Gageons que les petits paysans bretons ont dû trouver la culture scolaire française fort étrange quand l'école est devenue gratuite et obligatoire.

21 Ce qui est fascinant, c'est qu'au fond, les différences culturelles émergent de l'expérience scolaire plus qu'elles ne lui préexistent, sont produites par l'école plus qu'elles ne sont à l'origine des problèmes. Cela ne signifie évidemment pas que d'autres différences n'existent pas avant ou ailleurs, mais cela signifie plutôt que raisonner à partir d'un face-à-face universalisme / 
relativisme ne rend pas compte de ce qui se passe réellement et de façon complexe sur le terrain. En ce sens, l'école laïque d'aujourd'hui serait une fabuleuse machine à produire de la différence, du sujet. N'est ce pas un étrange paradoxe?

Je suis d'accord avec cette thèse des différences « réactives ». Le thème des différences émerge comme une des conséquences du sentiment d'injustice scolaire et, plus largement, des discriminations diverses auxquelles se heurtent les élèves tout au long de leur vie. Il surgit comme la mise à l'épreuve d'un sujet qui s'éprouve comme égal et découvre qu'il ne l'est pas. N'oublions pas que dans le monde scolaire les inégalités sociales et scolaires sont toujours beaucoup plus prégnantes que les inégalités / différences culturelles. Pour ce qui est de l'accusation de racisme portée contre certains enseignants, je me demande si elle n'est pas le plus souvent une rationalisation de l'échec, une manière de se libérer de la culpabilité de l'échec sachant que les élèves décrivent plus longuement et plus précisément les tensions « raciales » qui traversent les relations entre les élèves eux-mêmes et, plus encore, les relations entre les «bandes ».

Pour parler nettement, j'ai le sentiment qu'il existe aujourd'hui une très grande distance entre les expériences individuelles des acteurs d'une part, et les rhétoriques idéologiques et politiques qui se déploient à propos des migrants, de la laïcité, de la nation et de la République, d'autre part. Tout se passe comme si se construisait un problème collectif sur lequel se positionnent les partis politiques et les intellectuels, une rhétorique de la nation, de l'universalisme, du communautarisme, du postcolonialisme, etc., et comme si cette rhétorique collective écrasait l'expérience des individus sommés de s'y reconnaître. Dans l'enquête que j'évoquais plus haut, un nombre considérable de personnes nous disent ne pas reconnaître leur vie et leur expérience quand elles regardent la télévision où elles découvrent qu'elles sont un "problème ", une "menace ", une " communauté ", un ensemble de «victimes »... alors qu'elles ne se perçoivent pas ainsi. Le risque majeur est là: les catégories idéologiques et politiques écrasent l'expérience des acteurs instrumentalisés par leurs adversaires et parfois par leurs porte-paroles. En fait, les élèves d'origine étrangère et leurs parents sont, le plus souvent, des symptômes transformés en causes.

Il se creuse une grande distance entre les expériences individuelles des discriminations, expériences ambivalentes, complexes, souvent incertaines à l'exception de celles des jeunes garçons de banlieue qui se construisent dans cette discrimination, et la formation des représentations de la nation, des problèmes sociaux et de la culture qui attribuent aux migrants une place essentielle dans le dispositif symbolique d'explication de la crise du modèle de la société nationale. Toute chose inégale par ailleurs, cette situation n'est pas radicalement nouvelle: l'antisémitisme nazi appelant à la «solution finale" n'était certainement pas l'expression directe de l'antisémitisme des Allemands et de la situation des Juifs en Allemagne; il fut d'abord et avant tout une construction politique, une réponse "magique» aux tensions de la société allemande. Au sens littéral, cette comparaison avec la situation française actuelle est évidemment inacceptable et scandaleuse, mais je l'utilise malgré tout pour faire comprendre ce mécanisme de disjonction entre l'expérience des individus, ici celle des élèves et des maitres, et les constructions idéologico-politiques qui sont censées lui donner du sens. Il va de soi que c'est la disjonction elle-même qui est dangereuse puisque l'univers des représentations et des fantasmes "s'affole» et peut avoir des effets en retour catastrophiques sur les expériences individuelles. 

n'a guère donné de place aux établissements et à la formation d'ordres scolaires locaux, d'ordres négociés avec ce que les anglo-saxons appellent la «communauté ». La laïcité universaliste supposait que chaque établissement, chaque classe, chaque enseignement soit strictement identique à tous les autres. Même si ce cadre n'a jamais empêché les adaptations locales, il est un modèle supposant que les demandes sociales, celles des parents, des associations et des entreprises, soient tenues à distance. En fait cette école a été républicaine mais peu démocratique dans la mesure où rien ne pouvait y être discuté. C'est ce cadre-là de la laïcité qui s'épuise aujourd'hui avec ce que j'ai appelé le déclin du " programme institutionnel ».

L'école joue maintenant un rôle si décisif dans la vie des individus, et de tous, que l'on ne peut guère imaginer que ceux-ci en soient toujours des personnages muets. Aussi les demandes et les stratégies des uns et des autres se manifestent-elles de manière intense tout en n'ayant guère d'espace institutionnel d'expression. Derrière la neutralité et l'indifférence laïque républicaine, souvent devenues un slogan et un décor, se développe tout un marché scolaire largement implicite et hypocrite dont les principales victimes sont ceux qui ignorent qu'il s'agit là d'un marché. Les parents efficaces ne sont plus seulement ceux qui disposent des codes culturels les plus adéquats, ce sont aussi les stratèges les mieux avertis. Les établissements participent à ce "marché » et les élèves eux-mêmes développent des stratégies instrumentales et utilitaires parfaitement maitrisées pour les uns, inefficaces pour les plus faibles qui choisissent de décrocher silencieusement ou de se révolter à grand bruit. Les élèves "marchandent» leur autonomie derrière un deal faisant fonction de contrat éducatif : l'autonomie juvénile est contrôlée par les normes de l'efficacité scolaire. Deal qui ne vaut évidemment que pour les élèves des classes moyennes et qui s'effondre dans les catégories sociales les moins favorisées quand le succès n'est pas au rendez-vous.

Le modèle éducatif républicain (centré sur la transmission ritualisée de savoirs incontestables, la régularité des rites scolaires, l'autorité "sacrée » du maître, la formation d'un sanctuaire scolaire...) laisse la place aux imprécations nostalgiques pour ne pas dire réactionnaires et à des arrangements locaux, parfois réussis, mais, le plus souvent, aléatoires et fragiles car tenant à la personnalité et au talent des enseignants et à la solidité des équipes. Pour refonder un contrat éducatif, il faudrait être en mesure de dire quelles sont les normes, les règles, les devoirs et les droits des uns et des autres. Et ceci suppose deux choses. Il faudrait d'abord admettre que les autres existent, que les parents, les élèves, les associations qui parlent en leur nom, les employeurs potentiels... aient leur mot à dire, sans que l'école choisisse a priori ceux qu'elle accepte parce qu'ils se soumettent au modèle scolaire. Il faudrait ensuite que l'on soit capable de dire ce qui est discutable, négociable, et ce qui ne l'est pas. Ce qui revient à définir la laïcité puisqu'il va 
de soi que tout n'est pas négociable dans chaque établissement - à commencer par ce qui fonde le caractère commun de l'école puisqu'une école laïque publique n'existe que dans la mesure où elle est identifiée à ce que nous pouvons avoir de commun dans une société, fût-elle «pluriculturelle». Si l'on considère que les nations dans lesquelles nous vivons sont des "clubs », il faut en tracer les règles, les caractéristiques et la nature politique. C'est de cette manière que l'école républicaine pourrait devenir une école démocratique, c'est-à-dire une école dans laquelle on apprend à être un sujet démocratique, un individu singulier et le membre d'un collectif.

Sur le plan éducatif, notre école a connu une véritable régression. Si nous demandions aujourd'hui aux ministres et aux responsables syndicaux quel est le projet éducatif, « moral », de l'école, ils auraient beaucoup de mal à répondre à cette question en dehors de quelques clichés sur la citoyenneté, l'esprit critique et d'autres formules qui n'engagent plus guère. Notre vision de l'école est uniquement centrée sur son efficacité que savent les élèves, à quoi servent les diplômes... ? - et sur son équité pensée comme l'égalité des chances dans le recrutement des élites - combien de filles, d'enfants défavorisés et issus des minorités visibles accèdent aux grandes écoles? Il suffit de lire les programmes politiques et les déclarations syndicales pour voir que nous n'avons plus grand-chose à dire sur les qualités, les compétences cognitives et les « vertus » morales et sociales dont nous aimerions armer les élèves afin d'en faire les sujets de leur vie et les membres d'une société vivable. Dans ce domaine, l'école français semble parfois si écrasée par sa grandeur passée et par le fait qu'elle a été appelée à fonder la nation qu'elle est comme paralysée quand il s'agit de transformer les manières d'enseigner et de vivre dans l'école, de dire pratiquement quel citoyen elle veut former. D'ailleurs, les enquêtes internationales montrent que les élèves français sont les champions du pessimisme et de l'absence de confiance en soi et dans l'institution.

Dans ce contexte on comprend mieux la force du réflexe qui consiste à attribuer les problèmes de l'école aux élèves, à commencer par les plus pauvres et les plus "différents» d'entre eux, et à multiplier les dispositifs de soutien et les initiatives périscolaires qui permettraient de défendre le noyau dur d'un modèle scolaire dont on sait qu'il ne fonctionne que pour une moitié environ des élèves.

La confrontation de différentes formes de socialisations et identités particulières pose le problème de la légitimité du modèle laïque, unique et supérieur et questionne la laïcité dans ses possibilités de mise à jour, en référence au projet éducatif des Lumières. Votre défense d'une mise à jour de la laïcité est ambitieuse, dans la mesure où ce principe n'y serait alors pas réduit à un simple arbitrage des tensions scolaires par la garantie d'un ordre de neutralité, qui d'ailleurs n'existe pas, mais désignerait un enjeu d'accompagnement d'une expérience scolaire dont vous avez montré les dimensions de subjectivité, de stratégie et d'intégration. Vos analyses soulignent également l'hypocrisie et la facilité d'une position qui assimile les établissements d'élite et la méritocratie républicaine. Mais pensez-vous que la laïcité puisse être encore un projet? Pour certains, la laïcité républicaine ne peut plus être qu'un prétexte pour dicter la bonne façon d'être français et de faire société, dans le cadre d'un conflit de définition entre majoritaires et minoritaires. Et puis, vous avez montré que les élèves expriment souvent leur différence en termes de droit individuel, et non comme un principe, un droit culturel, communautaire. Ils revendiquent leurs tenues, leurs attitudes comme respectables, en tant que relevant de leurs propres choix. Tout cela n'est-il pas très difficile à concilier?

30 C'est parce que nous sommes confrontés à des différences sociales et culturelles que la laïcité reste à l'ordre du jour puisque nous devons vivre ensemble malgré ces différences 
et ces inégalités, ce qui suppose que nous ayons quelque chose de commun. Ce problème n'est peut-être pas plus difficile aujourd'hui qu'il l'était à la fin du XIXe siècle quand les religions semblaient inconciliables, quand les Lumières étaient combattues, quand les différences culturelles et linguistiques entre les classes sociales et les régions étaient bien plus fortes que nous le pensons aujourd'hui, quand la classe ouvrière était tenue hors de la cité. Dans ce contexte, la laïcité, c'était la tolérance plus la nation. Aujourd'hui, elle devrait être la tolérance plus la démocratie, c'est-à-dire la capacité de négocier la vie commune dès lors que la tolérance et l'égale liberté des individus ne sont pas « négociables ». Ceci suppose sans doute des "aménagements raisonnables » et, surtout, d'observer les faits et les conduites bien plus que les constructions dramatiques du déclin de la civilisation, de la culture et de la nation qui reviennent en force dans la rhétorique politique. Ceci suppose aussi que les acteurs dominés accèdent à la parole, qu'ils n'en soient plus privés soit par l'exclusion sociale et la relégation, soit par la captation de leur parole par des groupes autoproclamés.

Plutôt que de nous engager dans un psychodrame opposant la France laïque des Lumières à l'Islam obscurantiste et étranger (ou la France éternellement coloniale aux éternels descendants des esclaves), regardons les faits. Les familles populaires, musulmanes y compris, attendent plus de l'école que les familles de classes moyennes, elles y voient un espoir de salut culturel et social s'apparentant souvent à la foi du charbonnier. Or l'école se montre souvent peu accueillante pour ces familles, elle a souvent beaucoup de mal à les associer à son travail et plus souvent encore, elle ne parvient pas à faire réussir leurs enfants de manière acceptable. Plus exactement, si beaucoup de ces enfants réussissent, ceux qui échouent lourdement viennent presqu'essentiellement de ces milieux.

Quelle est la part de ceux qui exigent que l'on enseigne la Bible ou le Coran, quelle est la part de ceux qui refusent l'enseignement de la biologie comme étant contraire aux lois de Dieu? En posant les problèmes en ces termes, nous jouons à nous faire peur, et ceci d'autant plus que l'on ne voit pas quelle majorité, y compris issue de ce qu'on appelle « la diversité ", accepterait de renoncer à la science et à la distinction de la science et de la foi. Par ailleurs, il est toujours possible que les familles donnent à leurs enfants une éducation scolaire dans un cadre religieux affirmé, c'est le cas de nombreuses familles catholiques et, semble-t-il, de plus en plus de familles juives; personne ne pense que la République en soit menacée et rien n'interdirait que des familles musulmanes accèdent aux mêmes biens. Je crois donc nécessaire d'affirmer encore une fois qu'il existe une marge de discussions et d'aménagements de la tolérance laïque et qu'un grand nombre des débats qui nous occupent sont de pures productions politiques. Mais quand on ne se sent pas capable de régler les questions de la justice scolaire, peut-être a-t-on intérêt à affirmer qu'elles ne sont pas centrales et que le spectre communautariste nous menace.

On sait que les grands débats enflammés autour de la laïcité sont beaucoup moins tranchés sur le terrain, qu'il existe de modestes "accommodements raisonnables» et vos travaux montrent que l'école, hier comme aujourd'hui, aménage son universalisme au quotidien. De même, vous avez décrit la tolérance pratique et spontanée des enseignants; à l'école, la norme anti-raciste est forte et les sociabilités juvéniles ne recoupent pas les clivages ethniques.

Cependant, n'enregistre t-on pas aujourd'hui un certain durcissement de ton, à la fois du côté de l'État et du terrain? En avril 2011, le débat lancé par le gouvernement français sur la laïcité a suscité nombre de réactions et a donné lieu à 26 propositions. L'une d'entre elles propose d'« assurer une pédagogie de la laïcité » en mettant en place "une formation obligatoire à la laïcité à l'ensemble des agents des services publics (...), en développant «en lien avec les grands pôles 
universitaires un module de formation aux principes républicains et, spécialement, à la laïcité... ». Une deuxième proposition, pour "réaffirmer le principe de laïcité dans le cadre des services publics", envisage d'étendre par la voie législative "les exigences de neutralité et de laïcité des agents des services publics aux collaborateurs occasionnels du service public ». Il pourrait s'agir ainsi, encore plus loin que la loi de 2004, d'interdire, au nom de la laïcité, aux femmes portant un foulard d'accompagner la classe dans le cadre de sorties scolaires, régulières ou occasionnelles, comme la piscine, différents spectacles, les pique-niques. La mesure peut également concerner la participation aux conseils d'établissements, voire aux réunions parents-enseignants...

Encore une fois, j'ai le sentiment que l'on mélange tout. L'idée de former les fonctionnaires à la laïcité est sans doute excellente en principe, mais pour ce qui est des enseignants le besoin de formation professionnelle tout court est aujourd'hui très largement prioritaire. Il me semble que personne ne conteste vraiment le principe de neutralité religieuse des fonctionnaires dans l'école publique. Mais on voit bien que l'interdiction faite aux mères de famille portant foulard d'accompagner les enfants lors des sorties scolaires est une pure volonté d'exclure ; va-t-on faire la chasse aux médailles chrétiennes et aux étoiles de David, pour ne rien dire des gris-gris de tous ceux qui pensent ne pas avoir de religion?

Personne n'est dupe, le débat sur la laïcité et le débat sur la nationalité ont été des manières relativement "convenables" de désigner certains migrants et leurs enfants comme des dangers. Ce sont de pures opérations politiques profondément dangereuses car désignant des boucs émissaires et on peut se féliciter de leur faible succès et du fait que leurs conclusions n'ont rien dit de bien nouveau. En revanche, le déplacement de la question sociale vers la question raciale que l'on observe dans bien des pays d'Europe, y compris dans ceux qui en semblaient le mieux préservés, reste extrêmement dangereux, non seulement parce qu'il est moralement discutable, mais aussi parce qu'il ouvre un mécanisme de fractionnement infini ; les Nord contre les Sud, les Flamands contre les Wallons, les citoyens « intégrés et assimilés » d'origine nationale contre les citoyens tout aussi « intégrés et assimilés » d'origine étrangère... Il se développe un mécanisme général d'assignation aux racines et aux identités au moment où les racines et les identités sont plus aléatoires, multiples et flottantes qu'elles ne l'ont jamais été.

Malheureusement, il ne suffit pas de dénoncer les idées et les stratégies de ses adversaires politiques pour répondre aux problèmes qui se posent à nous. L'idée selon laquelle il n'y aurait que des problèmes sociaux, des problèmes de chômage et de pauvreté, l'idée aussi selon laquelle la coexistence des cultures serait fatalement harmonieuse peuvent être éminemment sympathiques, elles n'en sont pas moins fausses. De même, l'idée selon laquelle le refus de certaines caractéristiques culturelles ne serait qu'une forme d'ethnocentrisme postcolonial n'est crédible que dans les milieux très protégés des universités. Il ne suffit pas d'être victime d'injustice pour avoir toujours raison et le racisme et le sexisme des « racisés » ne sont pas plus acceptables que ceux des dominants même s'ils ont quelques excuses à faire valoir.

Il nous faut donc reprendre le projet de la laïcité scolaire et celui-ci me semble devoir être fondé sur le droit et la capacité des individus à construire leur expérience propre et leur singularité. Ce primat de l'individualisme n'est pas celui du narcissisme et de l'égoïsme. Pour être des sujets individuels, les acteurs ont besoin de cadres institutionnels, de protections juridiques, d'espaces politiques et de politiques sociales... Dans ce nouveau contexte, l'école laïque ne peut plus être le dernier temple de la République, de la nation 
et de la Raison placé hors du monde, elle doit être une école juste et accueillante, projet plus modeste et plus réaliste que les appels réitérés aux retours à un âge d'or scolaire.

La loi de 1882, sur la lä́cité, a supprimé l'enseignement religieux dans les écoles primaires publiques afin que tous les enfants puissent les fréquenter sans être obligés de pratiquer une religion jui pourrait ne pas convenir à eux ou à leurs parents. On respecte xinsi la liberté de conscience. La même loi de $188_{2}$ a rendu l'ins-

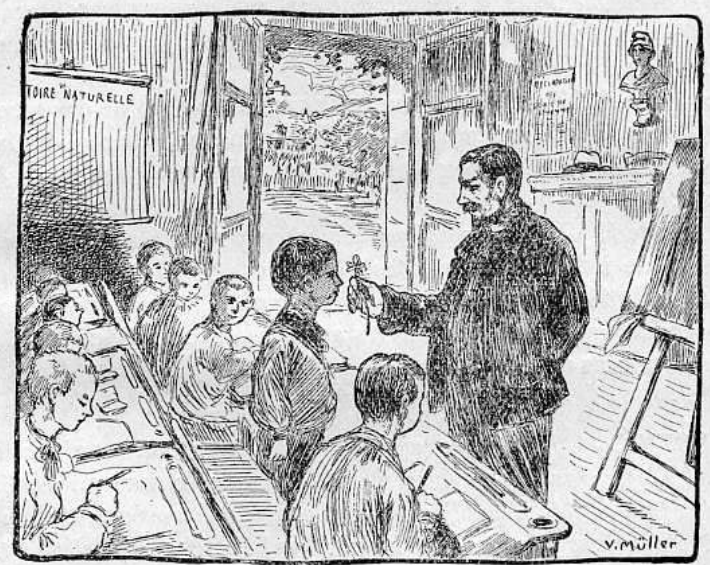

L'école primaire d'aujourd'hui

truction obligatoire pour tous les enfants de moins de treize ans. Grâce à ces lois, l'instruction du peuple a fait des progrès considérables. Avant la troisième République. 15 pour 100 des conscrits ne savaient ni lire ni écrire ; aujourd'hui, il n'y en a plus que 4 pour roo.

Histoire de la France expliquée aux enfants : Histoire de la nation et histoire de la civilisation, Cours élémentaire et moyen, G. DUVILLAGE (1936), Nouvelle édition, Bibliothèque d'éducation (Paris). Source CEDRHE : 10. p. 281

\section{BIBLIOGRAPHIE}

DUBET (François), Le Déclin de l'institution, Paris, éd. du SEUIL, 2002.

DUBET (François), Faits d'école, Paris, éd. de l'EHESS, 2008.

DUBET (François), Les Places et les Chances, Paris, éd. du SEUIL, 2010.

KYMLICKA (Will), La citoyenneté multiculturelle. Une théorie libérale du droit des minorités, Paris, La Découverte, 2001.

OGBU (John U.), « Les frontières culturelles et les enfants de minorités », Revue française de pédagogie, vol. 101, 1992.

VISIER (Laurent) et ZOÏA (Geneviève), La carte scolaire et le territoire urbain, Paris, PUF, 2009. 
INDEX

Keywords : education, secularism

Mots-clés : éducation, laïcité

\section{AUTEUR}

FRANÇOIS DUBET

Professeur de sociologie de l'université Bordeaux 2

Directeur d'études à l'École des hautes études en sciences sociales (EHESS) 